OPEN ACCESS

Edited by:

Bernd Mueller-Roeber,

University of Potsdam, Germany

Reviewed by:

Ashwani Pareek,

Jawaharlal Nehru University, India Ananda Mustafiz,

South Asian University, India

${ }^{*}$ Correspondence:

Daoyuan Zhang

zhangdy@ms.xjb.ac.cn

Specialty section:

This article was submitted to

Plant Abiotic Stress,

a section of the journal

Frontiers in Plant Science

Received: 09 November 2016 Accepted: 13 February 2017

Published: 27 February 2017

Citation:

Li X, Zhang D, Gao B, Liang $Y$,

Yang $H$, Wang $Y$ and Wood $A J$

(2017) Transcriptome-Wide

Identification, Classification, and Characterization of AP2/ERF Family Genes in the Desert Moss

Syntrichia caninervis.

Front. Plant Sci. 8:262.

doi: 10.3389/fpls.2017.00262

\section{Transcriptome-Wide Identification, Classification, and Characterization of AP2/ERF Family Genes in the Desert Moss Syntrichia caninervis}

\author{
Xiaoshuang Li', Daoyuan Zhang ${ }^{1 *}$, Bei Gao ${ }^{2}$, Yuqing Liang ${ }^{1,3}$, Honglan Yang ${ }^{1}$, \\ Yucheng Wang ${ }^{1}$ and Andrew J. Wood ${ }^{4}$
}

\begin{abstract}
${ }^{1}$ Key Laboratory of Biogeography and Bioresource in Arid Land, Xinjiang Institute of Ecology and Geography, Chinese Academy of Sciences, Urumai, China, ${ }^{2}$ State Key Laboratory of Agrobiotechnology, School of Life Sciences, The Chinese University of Hong Kong, Hong Kong, China, ${ }^{3}$ University of Chinese Academy of Sciences, Beijing, China, ${ }^{4}$ Department of Plant Biology, Southern Illinois University, Carbondale, IL, USA
\end{abstract}

APETALA2/Ethylene Responsive Factor (AP2/ERF) is a large family of plant transcription factors which play important roles in the control of plant metabolism and development as well as responses to various biotic and abiotic stresses. The desert moss Syntrichia caninervis, due to its robust and comprehensive stress tolerance, is a promising organism for the identification of stress-related genes. Using S. caninervis transcriptome data, 80 AP2/ERF unigenes were identified by HMM modeling and BLASTP searching. Based on the number of AP2 domains, multiple sequence alignment, motif analysis, and gene tree construction, ScAP2/ERF genes were classified into three main subfamilies (including 5 AP2 gene members, 72 ERF gene members, and 1 RAV member) and two Soloist members. We found that the ratio for each subfamily was constant between S. caninervis and the model moss Physcomitrella patens, however, as compared to the angiosperm Arabidopsis, the percentage of ERF subfamily members in both moss species were greatly expanded, while the members of the AP2 and RAV subfamilies were reduced accordingly. The amino acid composition of the AP2 domain of ScAP2/ERFs was conserved as compared with Arabidopsis. Interestingly, most of the identified DREB genes in $S$. caninervis belonged to the A-5 group which play important roles in stress responses and are rarely reported in the literature. Expression profile analysis of ScDREB genes showed different gene expression patterns under dehydration and rehydration; the majority of SCDREB genes demonstrated a stronger response to dehydration relative to rehydration indicating that ScDREB may play an important role in dehydrated moss tissues. To our knowledge, this is the first study to detail the identification and characterization of the AP2/ERF gene family in a desert moss. Further, this study will lay the foundation for further functional analysis of these genes, provide greater insight to the stress tolerance mechanisms in S. caninervis and provide a reference for AP2/ERF gene family classification in other moss species.

\section{Keywords: AP2/ERF genes, Syntrichia caninervis, transcriptome, classification, desiccation tolerance}

Abbreviations: DREB, dehydration-responsive element-binding protein; DT, desiccation tolerance; ERF, ethyleneresponsive factor; RAV, related to ABI3/VP1; RT-qPCR, Reverse transcription quantitative real-time polymerase chain reaction; TF, transcription factor. 


\section{INTRODUCTION}

Syntrichia caninervis is a dominant moss species of biological soil crusts in the Gurbantunggut desert of Northwestern China (Zhang, 2005). S. caninervis has gained increasing attention due to a comprehensive tolerance to stresses such as desiccation, elevated temperature, low temperature, and high radiation. Studies on $S$. caninervis have been focused on the morphology (Stark et al., 2004, 2005; Xu et al., 2009a; Zheng et al., 2011; Tao and Zhang, 2012; Pan et al., 2016), physiology (Xu et al., 2009b; Li et al., 2010; Zhang et al., 2011; Wu et al., 2012; Yin and Zhang, 2016), and response to DT. S. caninervis is being developed as a model moss for studying the molecular mechanisms of DT and a good plant material for identification of stress-related genes (Wood, 2007; Yang et al., 2012, 2015; Li et al., 2015).

APETALA2/Ethylene Responsive Factor (AP2/ERF) is a large family of plant TFs, containing at least one DNA-binding domain (AP2 domain), which play important roles in the control of primary metabolism, secondary metabolism, and development as well as response to various biotic and abiotic stresses (Licausi et al., 2013). Two main classification methods have been proposed for the plant AP2/ERF superfamily based upon sequence similarities and the number of AP2 domains. Sakuma et al. (2002) classified the AP2/ERF superfamily into five families: AP2, RAV, DREB, ERF, and Soloists. The ERF family is also known as the EREBP (ethylene-responsive element binding proteins) family (Nakano et al., 2006). The AP2 family contains two AP2 domains, the RAV family contains one AP2 domain and one B3 domain, while the DREB, ERF, and Soloists families each contain a single AP2 domain. Furthermore, according to sequence similarity of the single AP2 domain, DREB family genes are further classified into the groups A1 to A6 and ERF family genes are divided into the groups B1 to B6 (Sakuma et al., 2002). Nakano et al. (2006) classified AP2/ERF predicted amino acid sequences proteins into three major families: AP2, ERF (include both DREBs and ERFs), and RAV. The ERF family was sub-divided into 12 groups in Arabidopsis and fifteen groups in rice according to the structure and similarity of the AP2 domain (Nakano et al., 2006). Both classification schemes have been extensively employed in the literature, and the methods have direct correspondence. For example, the A-1 type of DREB family in Sakuma's classification corresponds to the Group III ERF family in Nakano's classification (Nakano et al., 2006).

APETALA2/Ethylene Responsive Factor superfamily genes and transcripts have been identified and characterized in many plants and the superfamily has been studied extensively in the context of plant stress tolerance (Xu et al., 2011; Mizoi et al., 2012). DREB family genes have been documented to respond to drought, desiccation, osmotic stress, salt, low temperature (cold) and elevated temperature (heat) and are candidate genes for improving plant stress tolerance in crop plants (Lata and Prasad, 2011). The genome-wide identification of the AP2/ERF gene family has been accomplished in a number of plant species including Arabidopsis (Czechowski et al., 2005), rice (Narsai et al., 2010), poplar (Zhuang et al., 2008), grape (Licausi et al., 2010), soybean (Zhang et al., 2008), castor bean (Xu et al.,
2013), cotton (Lei et al., 2016), lotus (Sun et al., 2014), Chinese cabbage (Song et al., 2013), Medicago truncatula (Shu et al., 2015), and Musa species (Lakhwani et al., 2016). Using transcriptome and EST data, AP2/ERF superfamily genes has been analyzed in Brassica ssp. (Zhuang et al., 2010; Zhuang and Zhu, 2014), Triticum aestivum (Zhuang et al., 2011), Hevea brasiliensis (Duan et al., 2013), and tea (Wu et al., 2015). Gao et al. (2014) generated the first large scale transcriptome dataset for $S$. caninervis consisting of 92,240 unigenes. In this study, we identified 80 $A P 2 / E R F$ genes within the $S$. caninervis transcriptome. Based upon phylogenetic and protein motif structural analyses, 80 members of the ScAP2/ERF family were classified using the Arabidopsis AP2/ERF superfamily classification as reference. The expression pattern of ScERF genes were analyzed using RTqPCR in response to a DT-plant specific dehydration-rehydration protocol employed by our research group (Li et al., 2015). This study is the first identification, classification, and characterization of the AP2/ERF gene family in a moss species. As such, it will lay the foundation for further functional analysis of these $A P 2 / E R F$ family genes and provide valuable information in understanding the molecular mechanisms of the stress response in S. caninervis.

\section{MATERIALS AND METHODS}

\section{Annotation of the AP2/ERF Protein Families in S. caninervis}

Previously, we established a comprehensive transcriptome of $S$. caninervis transcripts during a standardized dehydrationrehydration process (Gao et al., 2014). Ninety-two thousand, two hundred and forty $S$. caninervis unigenes were obtained and served as the source for the AP2/ERF genes identification presented in this study. Arabidopsis and Physcomitrella patens $A P 2 / E R F$ family genes were downloaded from the plant TF database (PlantTFDB v3.0) ${ }^{1}$ (Jin et al., 2014). The HMM profiles were downloaded from Pfam database 27.02 (Punta et al., 2012).

One hundred and seventy-six Arabidopsis AP2 predicted amino acid sequences and one hundred and seventy-one $P$. patens AP2 predicted amino acid sequences were used as queries to search against the $S$. caninervis transcriptome database using BLASTP programmer, and an $E$ value of $1 \mathrm{e}-3$ was adopted. Next, the HMM profile PF00847 (AP2 domain) and PF02362 (B3 domain) were queried using hmm search command included in the HMMER (v3.0) software with an $E$ value cutoff at $1 \mathrm{e}$ 3. All the candidate $S c A P 2 / E R F$ genes identified through these two methods were confirmed with Conserved Domain Database $\left(\mathrm{CDD}^{3}\right.$; Marchler-Bauer et al., 2015) and SMART ${ }^{4}$ (Letunic et al., 2015) search to ensure the presence of AP2 domain. An AP2 domain length of approximately 60 amino acids was considered to be a full-length AP2 domain (Duan et al., 2013). Only sequences longer than 200 nucleotides were retained for further analysis. Sequences which shared $>95 \%$ matches were considered

\footnotetext{
${ }^{1}$ http://planttfdb.cbi.pku.edu.cn/index.php

${ }^{2}$ http://pfam.sanger.ac.uk/

${ }^{3}$ http://www.ncbi.nlm.nih.gov/cdd/

${ }^{4}$ http://smart.embl-heidelberg.de/
} 
redundant. The detailed flowchart of AP2/ERF gene detection in S. caninervis is depicted in Supplementary Figure S1.

\section{Sequence Analysis and Phylogenetic Tree Construction}

Open reading frames (ORFs) were predicted with the ORF Finder at $\mathrm{NCBI}^{5}$. Motif detection was performed with the online tool MEME program $^{6}$ (Bailey et al., 2009) using the parameters: any number of repetitions per sequence, motif width ranges of 6-50 amino acids, and 11 as the maximum number of motif. The motif alignment was conducted using $\mathrm{MAST}^{7}$ and Patmatch programs ${ }^{8}$ (Bailey et al., 2009). Physical and chemical characterization of genes with deduced amino acid sequence were analyzed using ProtParam $^{9}$ and protein subcellular location(s) were predicted using PSORT ${ }^{10}$. Multiple sequence alignment was performed with ClustalW in conjunction with MEGA 5.1 (Tamura et al., 2011), phylogenetic trees were constructed by the neighbor-joining (NJ) method using MEGA 5.1 (Poisson correction and pairwise deletion). Support for nodes on the estimated phylogeny was tested with 1000 bootstrap replicates.

\section{Gene Expression Analysis in Dehydration-Rehydration Process}

\section{Primers for Real-Time PCR}

Reverse transcription quantitative real-time polymerase chain reaction primers were designed with Primer Premier 5.0. To verify primer specificity, the designed primer sets were searched using BLAST against the local transcriptional data of $S$. caninervis. The primer sets were further assessed using melting-curve analysis after RT-qPCR and gel electrophoresis analysis of the amplicons.

\section{Plant Material Treatment}

Syntrichia caninervis gametophytes were collected from the Gurbantunggut Desert of Xinjiang Uyghur Autonomous Region of China (Fukang County, $44^{\circ} 32^{\prime} 30^{\prime \prime} \mathrm{N}, 88^{\circ} 6^{\prime} 42^{\prime \prime} \mathrm{E}$ ) as described by Li et al. (2015). For the desiccation-rehydration process, dry gametophores were fully hydrated with MINIQ-filtered water for $24 \mathrm{~h}$ (which served as the control), followed by desiccation at room temperature in glass desiccators (using activated silica gel as desiccant) at $25^{\circ} \mathrm{C}$ (Yang et al., 2012). Samples were collected after $0.5,2,6,12$, and $24 \mathrm{~h}$ of dehydration. Samples were subsequently rehydrated by transferring the plants to new Petri plates and the filter paper was saturated with $8 \mathrm{~mL}$ filtered water at $25^{\circ} \mathrm{C}$; hydrated samples were harvested after $0.5,2,6,12$, and $24 \mathrm{~h}$ rehydration.

\section{RNA Extract and cDNA Synthesis}

Total RNA was extracted using RNAiso reagent (Takara, Japan). Genomic DNA contamination was eliminated using RNase-free

\footnotetext{
${ }^{5} \mathrm{http} / / /$ www.ncbi.nlm.nih.gov/gorf/gorf.html

${ }^{6} \mathrm{http}: / /$ meme-suite.org/index.html

${ }^{7} \mathrm{http}: / /$ meme-suite.org//tools/mast

${ }^{8}$ https://www.arabidopsis.org/cgi-bin/patmatch/nph-patmatch.pl

${ }^{9} \mathrm{http}: / /$ web.expasy.org/protparam/

${ }^{10} \mathrm{http}: / /$ psort.hgc.jp/form.html
}

DNaseI (Takara, Japan). RNA quality was determined using gel electrophoresis and a NanoDrop ND-2000 spectrophotometer (Thermo Fisher Scientific, USA). The 260/280 ratio of RNA samples between 1.8 and 2.1, and 260/230 ratio higher than 1.8 were used for subsequent experiment. First strand cDNA was synthesized using PrimeScript ${ }^{\mathrm{TM}}$ RT reagent kit (Takara, Japan) according to the instruction. cDNAs qualities were tested as templates by amplifying the ScACT gene using RT-PCR (data not shown). All cDNA was stored at $-20^{\circ} \mathrm{C}$ prior to use.

\section{RT-qPCR Assay and Data Analysis}

RT-qPCR reactions were carried out using CFX96 Real-Time PCR Detection System (Bio-Rad, USA) and SYBR Premix Ex $\mathrm{Taq}^{\mathrm{TM}}$ kit (Takara, Japan). The reaction mixture consisted of $2 \mu \mathrm{l}$ 1:5 diluted cDNA samples, $0.4 \mu \mathrm{l}$ each of the forward and reverse primers $(10 \mu \mathrm{M}), 10 \mu \mathrm{l}$ real-time master mix, and $7.2 \mu \mathrm{l}$ water in a final volume of $20 \mu \mathrm{l}$. Three biological replicates and three technical replicates of each biological replicate with a no-template control (NTC) were also used. The RT-qPCR protocol was as follows: $30 \mathrm{~s}$ initial denaturation at $95^{\circ} \mathrm{C}, 40$ cycles of $94^{\circ} \mathrm{C}$ for $5 \mathrm{~s}$, and $58-60^{\circ} \mathrm{C}$ for $30 \mathrm{~s}$. The relative expression levels of genes were calculated relative to the control (fully hydrated samples). The combination of ScACT and Sca-TUB are used to reliably normalize the RT-qPCR data based upon our previous research (Li et al., 2015).

\section{Statistical Analysis}

Statistical analyses were performed using SPSS software (standard version 11.5 released for Windows, SPSS Inc., Chicago, IL, USA). All data were analyzed using a one-way analysis of variance (ANOVA) at the 95\% confidence level. The significant difference was compared using LSD multiple comparison test. The data shown are the mean values $\pm \mathrm{SE}$ of three replicates, and the significance level relative to controls is ${ }^{*} P<0.05,{ }^{* *} P<0.01$. Figures were generated using Sigmaplot10.0. Adobe Illustrator CS5 and Adobe Photoshop CS3 were used for image processing.

\section{RESULTS}

\section{Identification and Classification of AP2/ERF Genes}

Eighty AP2/ERF unigenes were identified from the $S$. caninervis transcriptome using HMMs and BLASTP (see Supplementary Figure S1 for the detailed screening strategy). The unigenes ranged from 202 to $2514 \mathrm{bp}$ in length, and the corresponding predicted amino acid sequences ranged from 39 to 659 aa. AP2 domain prediction of the ScAP2/ERF genes demonstrated that 68 of the 80 unigenes (85\%) contained a full-length AP2 domain (ca $60 \mathrm{aa}$ ), five unigenes contained two AP2 domains, and a single unigene contained both a single AP2 domain and a B3 domain. Seventeen of the 80 unigenes (21\%) contained an intact ORF. Based on these results, the 74 unigenes encoding a single AP2 domain which were preliminarily classified as members of the ERF protein family (some special AP2 family protein members and Soloist proteins also contain a single 
AP2 domain). The five AP2 double-domain unigenes were classified as AP2 protein family members and the AP2/B3 unigene was classified as a RAV protein family member. Group classification of the $S c A P 2 / E R F$ genes was accomplished by constructing a phylogenetic gene tree which aligned the predicted AP2 domains using the NJ method. Nine unigenes were excluded from further analysis due to truncated AP2 domains, finally 71 predicted amino acid sequences were used to construct the phylogenetic tree. The un-rooted gene tree demonstrated that the $71 \mathrm{~S}$. caninervis AP2/ERF predicted amino acid sequences can be divided into five main groups: ERF I, ERF II, ERF III, AP2, and RAV (Supplementary Figure S2).

Sixty-five unigenes containing a single AP2 domain grouped into three clades: ERF I (two sequences), ERF II (41 sequences), and ERF III (22 sequences). Five unigenes which contained two AP2 domains grouped into the same clade (AP2), and the AP2/B3 unigene formed a unique clade (RAV). Additional phylogenetic analysis employing $71 \mathrm{ScAP2/ERF}$ predicted amino acid sequences and 176 previously annotated Arabidopsis AP2/ERF predicted amino acid sequences supported the classification into five groups (Figure 1). The number of ScAP2/ERF subfamily genes were compared with the model plants Arabidopsis and $P$. patens. The ERF subfamily was the largest in each species ranging from 77 to $90 \%$ (Table 1), while RAV and Soloist subfamily was the smallest ranging from 1 to $4 \%$.

\section{Classification of ScERF Genes}

Within the plant AP2/ERF family, the ERF subfamily is dominant and has been extensively documented to play important roles in plant responses to biotic and abiotic stresses (Zhuang et al., 2008; Huang et al., 2016). Phylogenetic analysis using 63 ScERFs and 136 AtERFs (both of which excluded soloists) identified members of eight out of 12 subfamilies based upon the classification of Sakuma et al. (2002): A2, A3, A4, A6, B1, B3, B4, and B6. ScERF predicted amino acid sequences contained no members of the A1, A4, B2, and B5 subfamilies

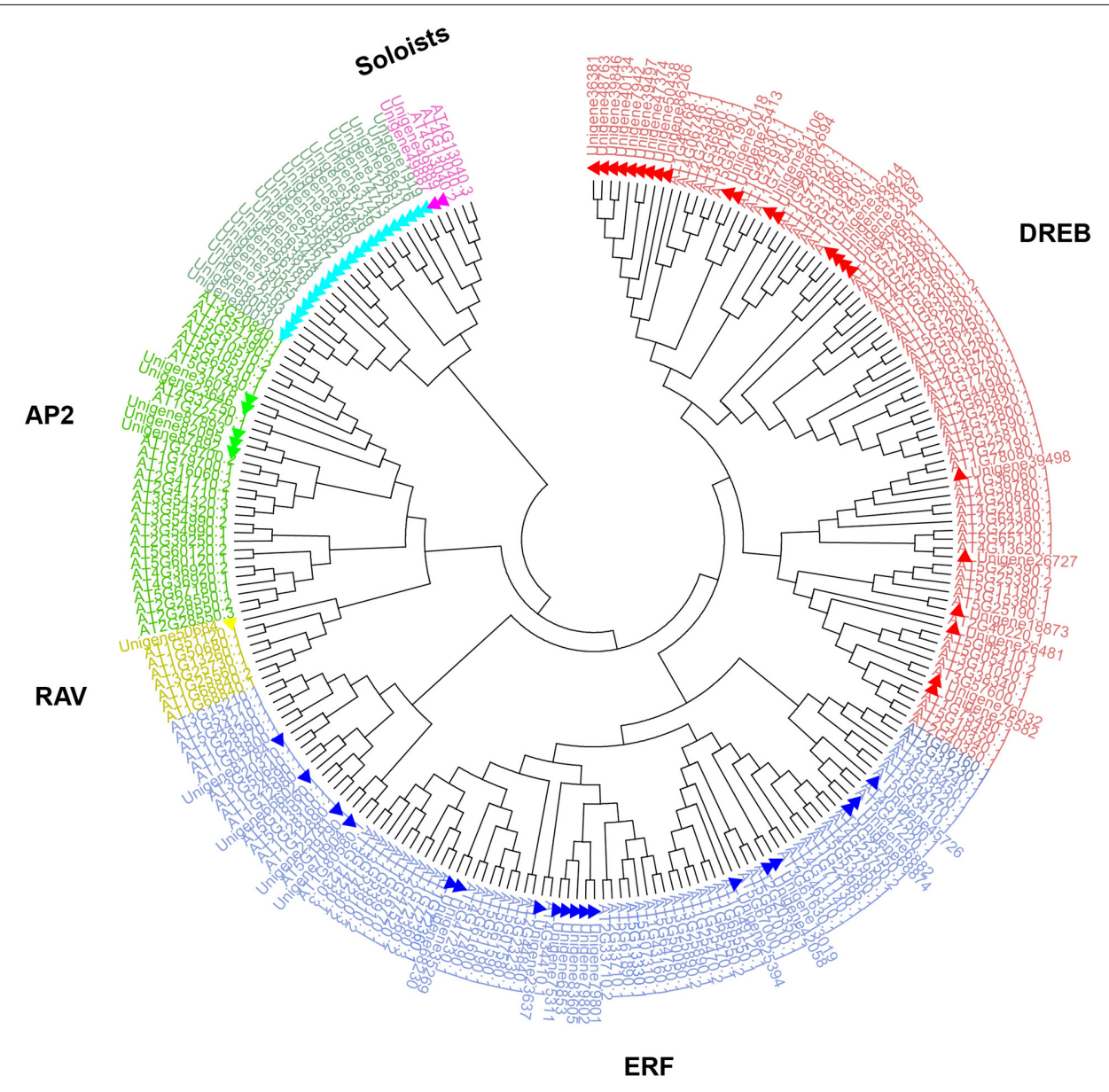

FIGURE 1 | Phylogenetic analysis of AP2/ERF family genes in Syntrichia caninervis and Arabidopsis. The gene tree was constructed using the neighbor-joining (NJ) method using 71 ScAP2/ERFs and 176 AtAP2/ERFs, Poisson model with pairwise deletion. Bootstrap values from 1000 replicates were indicated on the side of the node and used to assess the robustness of the tree. To distinguish ScAP2/ERFs from AtAP2/ERFs, ScAP2/ERF were marked with triangles. Different subfamily genes were marked and grouped in different colors (AP2, ERF, DREB, RAV, and Soloists subfamilies were labeled in green, blue, red, yellow, and purple, respectively). 
TABLE 1 | Distribution of APETALA2 (AP2)/ERF genes in Arabidopsis, Physcomitrella patens, and Syntrichia caninervis.

\begin{tabular}{|c|c|c|c|c|c|c|}
\hline \multirow[t]{2}{*}{ Subfamily } & \multicolumn{2}{|c|}{ A. thaliana genome } & \multicolumn{2}{|c|}{ P. patens genome } & \multicolumn{2}{|c|}{ S. caninervis transcriptome } \\
\hline & Number & Ratio & Number & Ratio & Number & Ratio \\
\hline AP2 subfamily & 30 & $17 \%$ & 13 & $8 \%$ & 5 & $6 \%$ \\
\hline ERF subfamily & 136 & $77 \%$ & 154 & $90 \%$ & 72 & $90 \%$ \\
\hline RAV subfamily & 7 & $4 \%$ & 2 & $1 \%$ & 1 & $1 \%$ \\
\hline Soloist & 3 & $2 \%$ & 2 & $1 \%$ & 2 & $3 \%$ \\
\hline Total & 176 & & 171 & & 80 & \\
\hline
\end{tabular}

Gene numbers of each subfamily conform to the PlantTFDB database (V3.0).

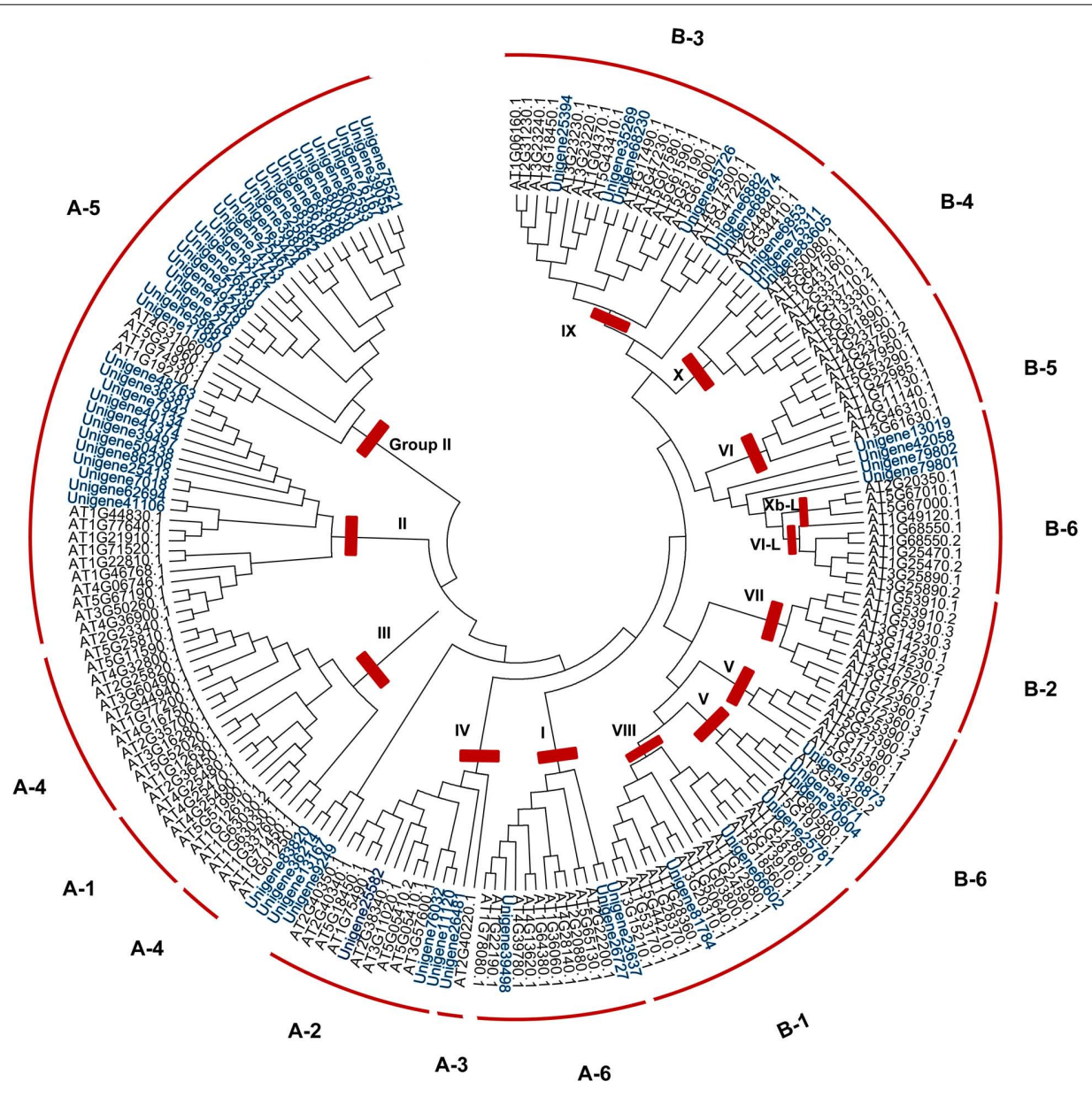

FIGURE 2 | Phylogenetic analysis of ERF family genes in S. caninervis and Arabidopsis. The gene tree was constructed using the NJ method using 63 ScERFs and 136 AtERFs, Poisson model with pairwise deletion. To assess the robustness of the tree, bootstrap values from 1000 replicates were indicated on the side of the node. To distinguish ScERFs from AtERFs, ScERF were marked in blue. Previously reported subfamily names (A1-6, B1-6) and group names (group I-Xb-L) were employed (Sakuma et al., 2002; Nakano et al., 2006).

(Figure 2 and Supplementary Figure S3). Accordingly, based upon Nakano et al. (2006)'s classification method, ScERF genes contained members of group I to group $\mathrm{Xb}-\mathrm{L}$, with the exception of group II, III and group VI. Most plants have a single A3 type DREB (Sakuma et al., 2002; Zhang et al., 2008) while some species (such as grape) lack A-3 type of DREBs (Licausi et al., 2010). Unigene26481 clustered together with both A-2 and A-3 type of AtDREBs (Figure 2) which prevented a definitive classification. Additional analysis using 57 AtDREBs generated a tree that showed unigene26481 was clearly grouped together with the A-3 type DREB in Arabidopsis (Supplementary Figure S3). $S$. caninervis has a single A-3 DREB protein. Interestingly, more than half of the ERF sequences are members of the A-5 subgroup DREBs in $S$. caninervis while other subgroups contain between two and six sequences (Figure 2 and Supplementary Figure S3). 


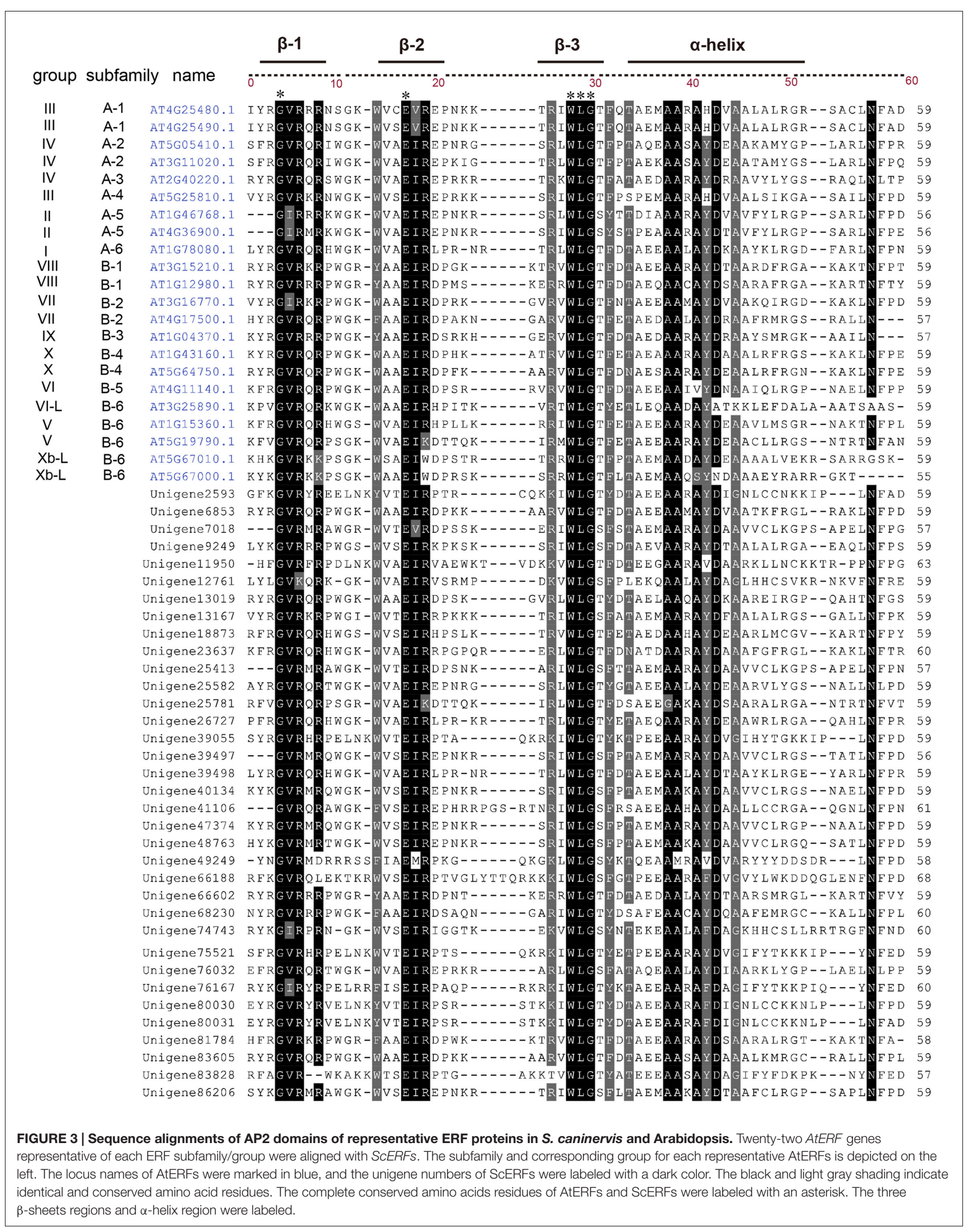


A

$\square$ motif $1 \square$ motif $2 \square$ motif3 $\square$ motif $4 \square$ motif5 $\square$ motif6 $\square$ motif7 $\square$ motif 8 $\square$ motif9 $\square$ motif 10 $\square$ motif 11

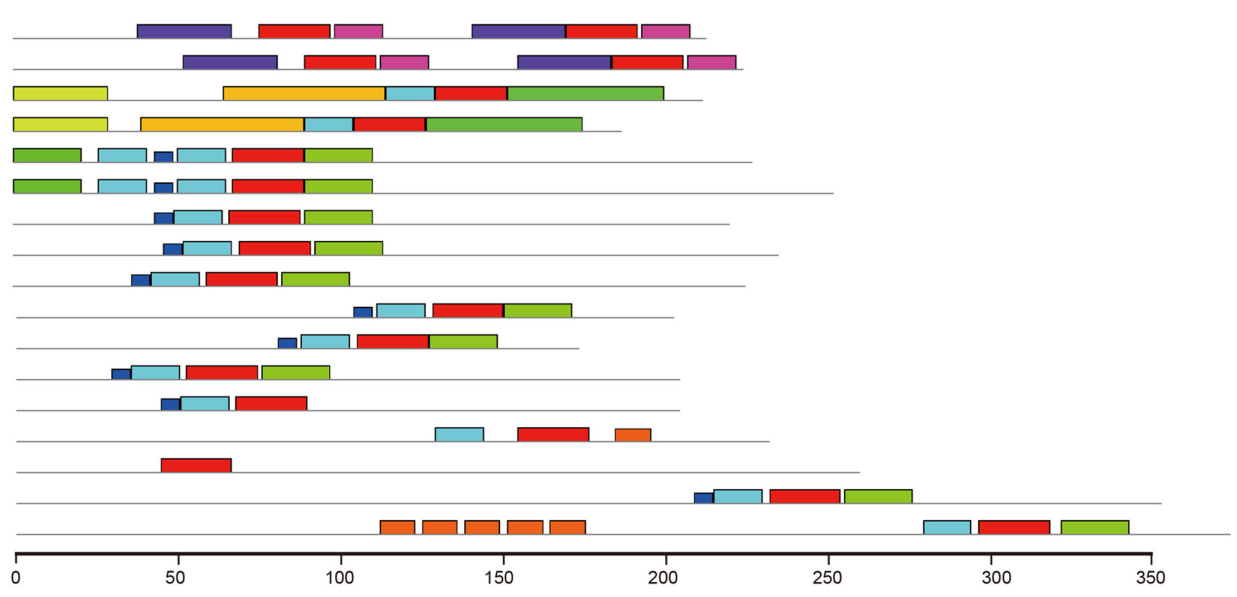

$\mathbf{B}$

name p-value

Unigene87894 $5.53 \mathrm{e}-55$

Unigene82095 $\quad 1.35 \mathrm{e}-54$

Unigene49887 $\quad 8.70 \mathrm{e}-16$

Unigene49889 2.26e-120

Unigene80030 $\quad 4.38 \mathrm{e}-65$

Unigene80031 3.14e-62

Unigene86206 $\quad 9.61 \mathrm{e}-43$

Unigene48763 3.79e-44

Unigene40134 7.16e-42

Unigene $75521 \quad 8.73 \mathrm{e}-38$

Unigene39055 $3.27 \mathrm{e}-40$

Unigene $9249 \quad 2.72 \mathrm{e}-31$

Unigene7018 2.00e-23

Unigene26831 3.23e-25

Unigene $83822 \quad 1.29 \mathrm{e}-10$

Unigene $83605 \quad 8.58 \mathrm{e}-33$

Unigene74743 $3.80 \mathrm{e}-38$

$$
0
$$

motif 1

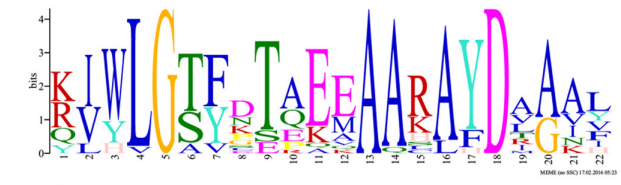

monts

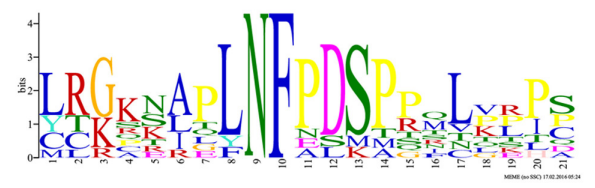

motif 5

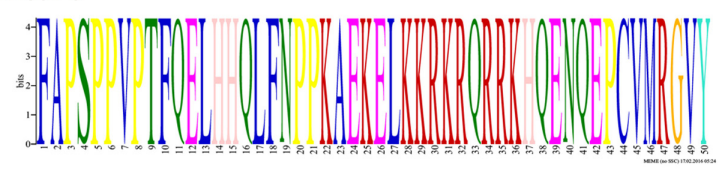

motif 7

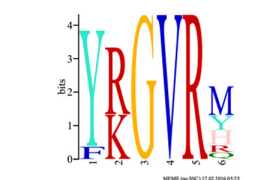

motif 10

motif 8

motif 2

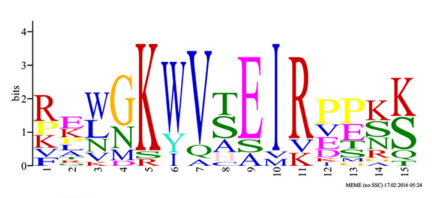

motif 4
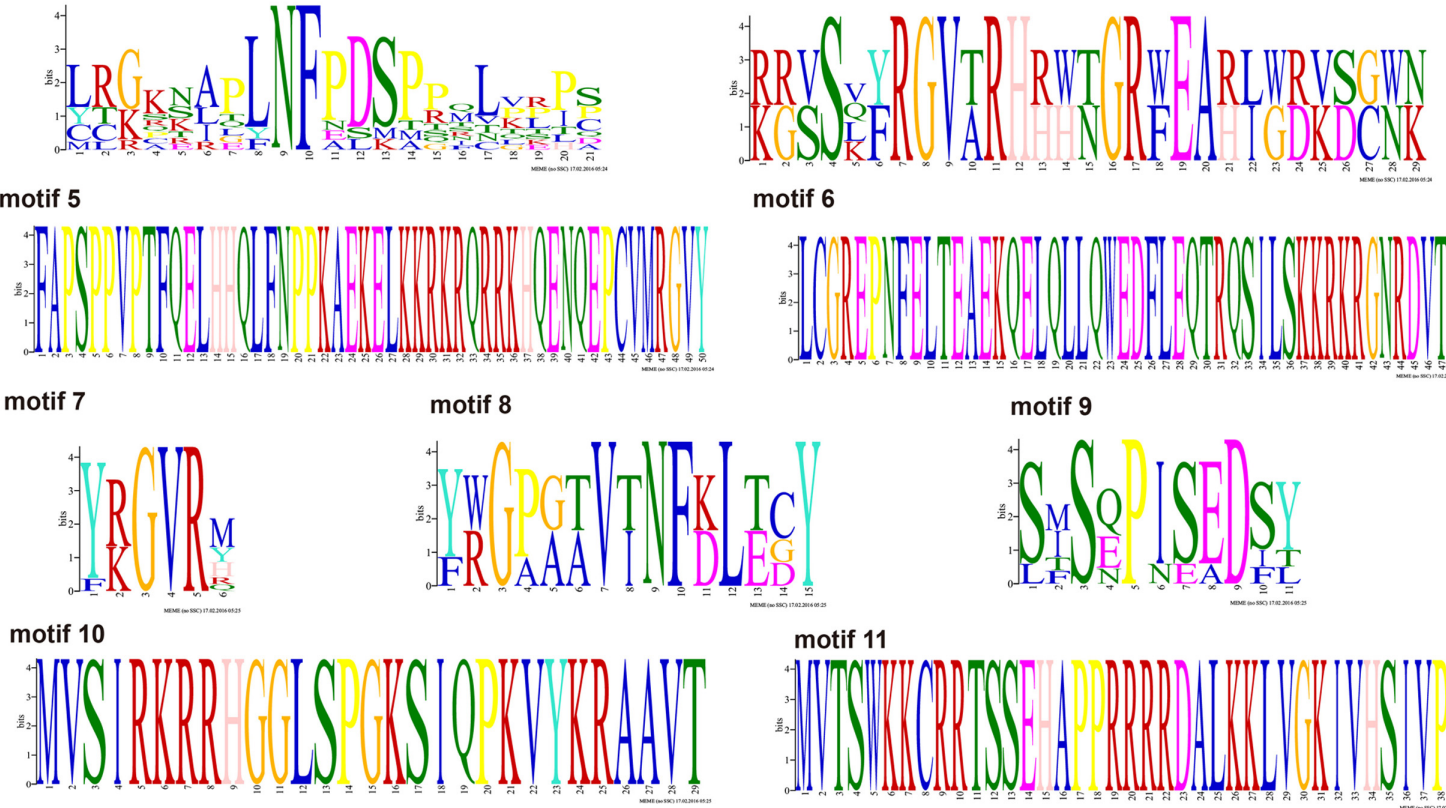

motif 6

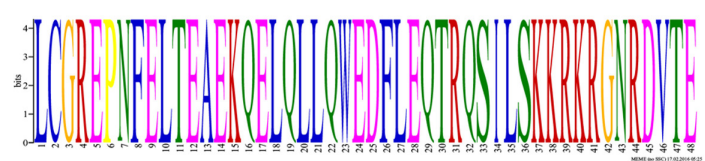

motif 9
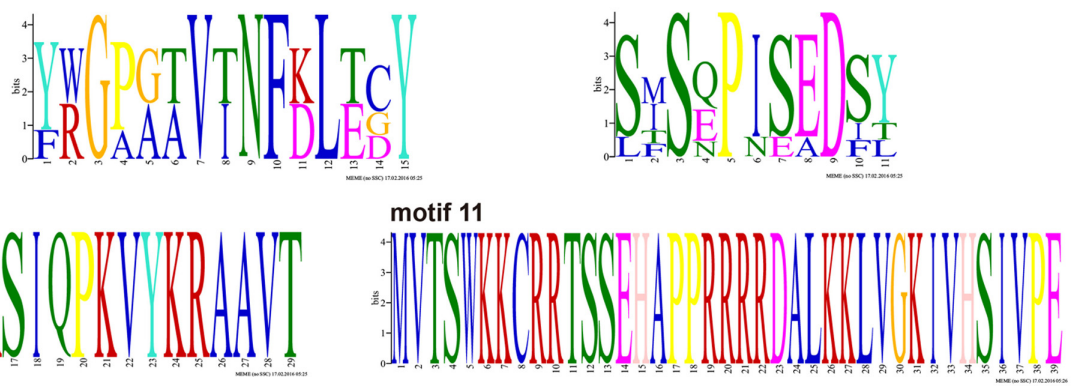

FIGURE 4 | Motif analyses of ScAP2/ERF with intact Open reading frames (ORFs) using MEME online software. Seventeen ScAP2/ERF proteins with complete ORFs were used for motif prediction. Parameters are as follows: any number of repetitions per sequence, motif width ranges of $6-50$ amino acids, and 11 as the maximum number of motif. Each of the sequence has an E-value less than 10. (A) Motif composition of ScAP2/ERF proteins, (B) deduced amino acid sequence of each motif.

\section{Conserved Amino Acid Residues in the AP2 Domain of ScERF Subfamily}

To analyze the conservation of the AP2 domains, ScERF deduced amino acid sequences were aligned with 22 AtERF sequences representing each ERF subfamily/group. Sequence alignment demonstrated that ScERF sequences shared significant amino acid similarity with AtERFs and that the AP2 domains of ScERFs also contained three $\beta$-sheets and one $\alpha$-helix (Figure 3 ). The amino acid residues 4 G, 16 E, 27 W, 28 L, 29 G were completely conserved in all ScERF and AtERF genes (labeled with asterisk). 
TABLE 2 | ScAP2/ERF deduced amino acid sequence characteristics and predicted subcellular location of genes.

\begin{tabular}{|c|c|c|c|c|}
\hline Unigene number & ORF length (aa) & Molecular weight (kDa) & Theoretical pl & Location \\
\hline$\underline{82095}$ & 223 & 25.50 & 9.58 & Nucleus (0.880) \\
\hline$\underline{87894}$ & 212 & 24.22 & 9.74 & Nucleus (0.76) \\
\hline 49887 & 186 & 21.60 & 10.07 & Nucleus (0.932) \\
\hline 49889 & 211 & 24.38 & 10.01 & Plasma membrane (0.79) \\
\hline 7018 & 203 & 22.23 & 5.17 & Microbody (0.558) \\
\hline 9249 & 203 & 22.30 & 5.97 & Nucleus (0.98) \\
\hline 26831 & 230 & 25.98 & 8.58 & Nucleus (0.76) \\
\hline 39055 & 172 & 19.13 & 9.16 & Nucleus (0.3) \\
\hline 40134 & 224 & 24.12 & 5.58 & Cytoplasm (0.45) \\
\hline 48763 & 234 & 25.04 & 5.8 & Cytoplasm (0.45) \\
\hline 75311 & 266 & 28.77 & 5.12 & Mitochondrial (0.467) \\
\hline 75521 & 201 & 22.46 & 9.58 & Cytoplasm (0.45) \\
\hline 74743 & 371 & 42.27 & 5.58 & Nucleus (0.70) \\
\hline 80030 & 251 & 27.78 & 5.93 & Nucleus (0.88) \\
\hline 80031 & 226 & 25.18 & 8.6 & Nucleus (0.867) \\
\hline 83605 & 350 & 37.82 & 5.30 & Nucleus (0.30) \\
\hline 83822 & 258 & 29.41 & 4.62 & Cytoplasm (0.45) \\
\hline 86206 & 219 & 24.04 & 6.45 & Nucleus (0.94) \\
\hline
\end{tabular}

APETALA2 genes were underlined, other genes were ERFS and Soloists.

$\beta$-sheet $1(\beta-1)$ contains the conserved GVR element (4G, 5V, $6 \mathrm{R})$ with several sequences substituting $5 \mathrm{I}$ for $5 \mathrm{~V}$. $\beta$-sheet 2 $(\beta-2)$ contains the conserved EIR element (16 E, $17 \mathrm{I}$, and $18 \mathrm{R})$. We found that in both Arabidopsis and S. caninervis, the amino acid residues EVR were observed only in A-1 group DREB genes, and the residues ERK were observed only in the B-6 protein subfamily (Figure 4). These conserved amino acid patterns may be helpful for the classification of ERF genes in other species. In addition, the unigene 49249 in S. caninervis had a particular "EMR" element in this position which was not found in Arabidopsis ERF genes. $\beta$-sheet $3(\beta-3)$ contained the conserved WLG element (27 W, 28 L, $29 \mathrm{G})$ which was highly conserved between Arabidopsis and S. caninervis, and the $\alpha$-helix contained a consensus sequence $\mathrm{AAxAxD}$ which was conserved between Arabidopsis and S. caninervis (Figure 3 and Supplementary Figure S4).

\section{Physicochemical Property, Conserved Motif, and Phylogenetic Analyses of ScAP2/ERF Proteins with Intact ORF}

Seventeen out of 80 ScAP2/ERF genes (2 ScAP2, 2 ScSoloists, and $13 S c E R F s$ ) were predicted to encode a single, intact ORF. The predicted polypeptide was characterized and the putative subcellular localization was analyzed (Table 2). The 17 ScAP2/ERF predicted polypeptides ranged from 172-to371 aa residues. The predicated molecular mass of the deduced polypeptides ranged from 19.13 to $42.27 \mathrm{KDa}$, and the theoretical pI values ranged from 4.62 to 10.0. More than half of ScAP2/ERF deduced polypeptides were predicted to localize in the nucleus (including two ScAP2). Four deduced polypeptides localized to the cytoplasm and one each localized to the microbody and plasma membrane. The $17 S c A P 2 / E R F$ predicted ORFs were submitted to MEME analysis to investigate the motif composition of the deduced polypeptides. In total, 11 motifs were found and their amino acids sequences were showed in Figure 4B. Five out of eleven motifs (motif $1,2,3,7,8$ ) represented the AP2 domain. Motif 2 and 7 correspond to the AP2 domain $\beta 2$-and $\beta 1$-sheet of AP2 domain, respectively, and Motif 1 contained the $\beta 3$-sheet and $\alpha$-helix. Motif 3 was similar to Motif 8 which corresponded to the C-terminal of the AP2 domain (Figure 4). ScERF genes with intact ORF should be the priority candidates for gene cloning and function analysis. Therefore, we constructed the gene tree using 15 ScERF genes (including two Soloists) with intact ORFs and 139 Arabidopsis ERF genes to establish a detailed classification. The tree revealed that $15 \mathrm{ScERF}$ s covered two ScSoloists, 12 A-5 type of DREBs, and one B-4 type of ERF gene (Figure 5). Three genes (unigene 40134, 48763, and 86206) belonged to A-5a subgroup, two genes (unigene 9249 and 7018) belonged to A-5b subgroup, and unigene 74743 which had the longest ORF belonged to A-5c subgroup. Additionally, six other A-5 type ScERF genes cannot be classified into a subgroup a, b nor c, which separately grouped in a S. caninervis-specific clade. One gene (unigene83605) was classified into the B-4 group.

\section{Expression Profiles of ScDREB Genes Response to Dehydration-Rehydration Process}

Based on the phylogenetic tree, eight ScDREBs representative of different group (such as A-5a, A-5b, A-5c) were selected to quantify the changes in transcript abundance during the dehydration-rehydration process using RT-qPCR analysis. RTqPCR results showed that compared to full-hydration (labeled as $0 \mathrm{~h}$ ), all eight transcripts accumulated both during the fast dry (FD) and rehydration (FDR) process (Figure 6). Under FD treatment, transcript abundance peaked at $6 \mathrm{~h}$ and then decreased, with the exception of unigene 7018 which peaked 


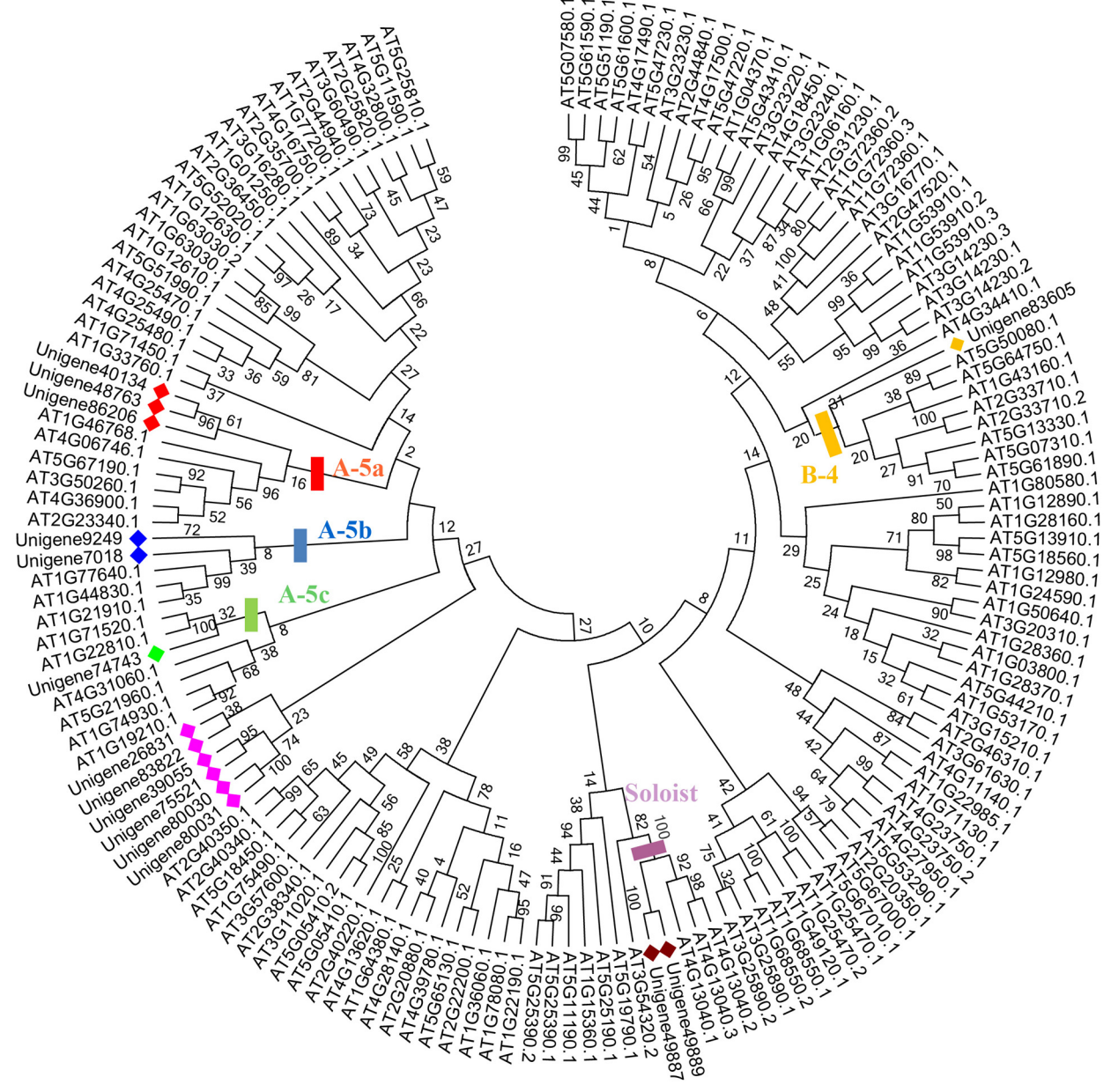

FIGURE 5 | Phylogenetic analyses of 15 ScERF genes with intact ORFs. The gene tree was constructed using the NJ method using 15 ScERFs with full-length ORFs and 139 AtERFs (including Soloist genes), Poisson model with pairwise deletion. Bootstrap values from 1000 replicates were indicated on the side of the node. ScERF were marked with diamonds using different colors to distinguish from AtERFs. Different clades represented specific groups were labeled with rectangles using the corresponding colors. A-5a, b, c group proteins were labeled with red, blue, and green, B-4 and Soloist groups were labeled with yellow and purple. Eight ScERF genes which cannot further classified were marked in pink.

at $12 \mathrm{~h}$ (Figure 6). During FDR process, transcript abundance increased at $0.5 \mathrm{~h}$ and subsequently decreased, with the exception of unigenes 80030 and 9249. The majority of the tested genes have higher transcript abundance during FD compared with FDR. For example the expression level of unigene 9249 increased 12 -fold at FD $6 \mathrm{~h}$, but was less than 4-fold increased during rehydration, suggesting that these genes may play a dominant role in fast drying process. While for unigene 40134, which has higher expression level during rehydration process, the gene expression level increased up to 12 -fold at FDR $0.5 \mathrm{~h}$ compared to the control, suggesting unigene 40134 may play important roles at the early stage of rehydration.

\section{DISCUSSION}

The AP2/ERF genes has been identified in many plant species due to its important roles in development, metabolism, and in response to various stresses (Mizoi et al., 2012; Licausi et al., 2013). AP2/ERF classification employs a well-established nomenclature established in Arabidopsis and rice (Nakano et al., 2006) and this nomenclature has been used to classify the AP2/ERF family genes in other plant species (Mizoi et al., 2012; Song et al., 2013). Advancements in sequencing technology and the availability of large datasets has aided the identification of $A P 2 / E R F$ family genes in several plant species using both genomic, transcriptomic or EST data (Licausi et al., 2010; Duan et al., 2013; Zhuang and Zhu, 2014; Wu et al., 2015; Lei et al., 2016). In the model moss $P$. patens $A P 2 / E R F$ s were demonstrated to be regulated in response to salinity, UV and other stresses indicating that AP2/ERF transcriptional factors also play a central regulatory role during stress response in moss species (Hiss et al., 2014).

Syntrichia ruralis exhibits comprehensive stress tolerance (Oliver et al., 1993; Zhang et al., 2009), and has been a good resource for identifying of genes associated with stress tolerance 

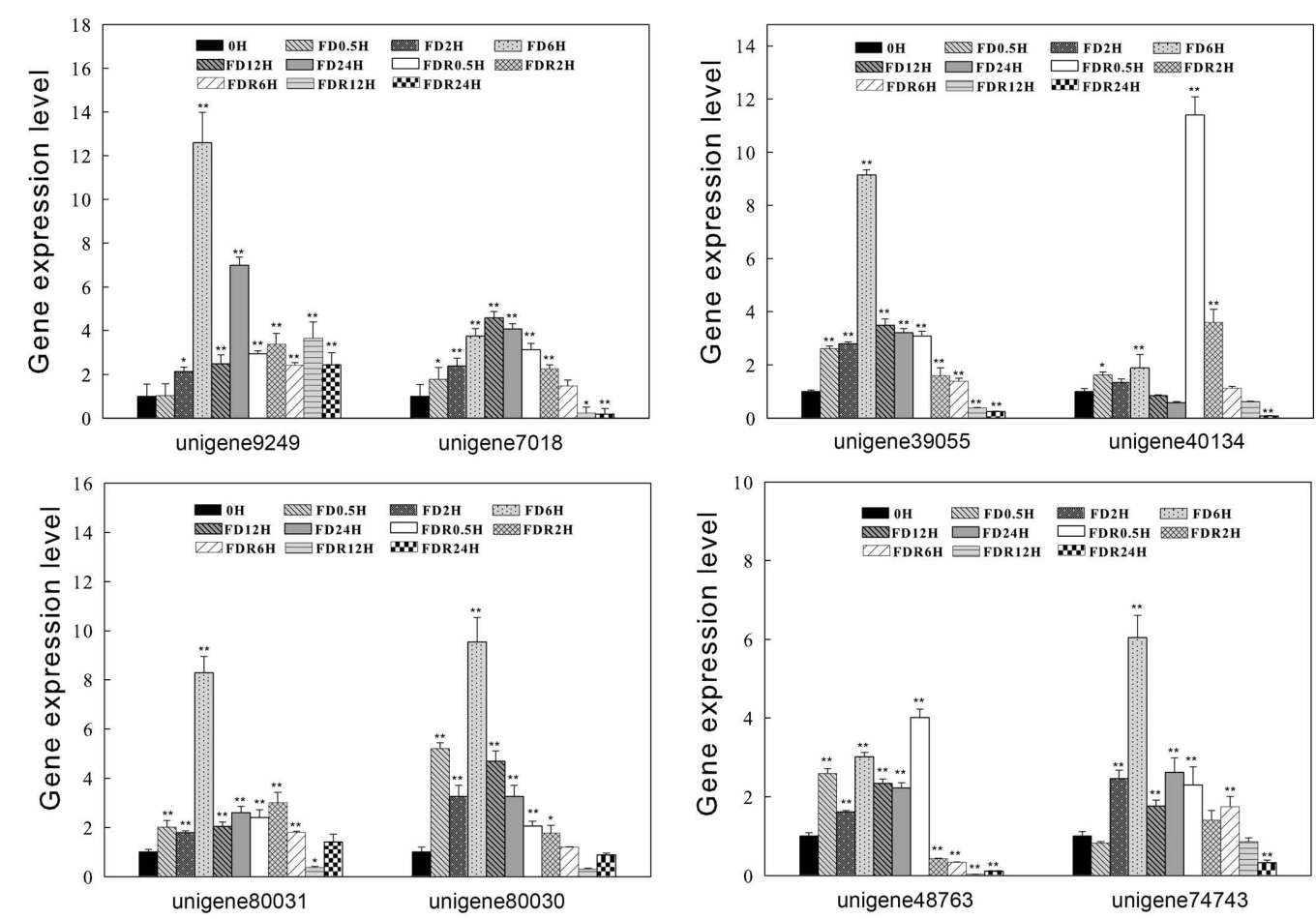

FIGURE 6 | Expression profiles of eight ScDREB genes during S. caninervis dehydration-rehydration process. The relative gene expression levels were calculated relative to $0 \mathrm{~h}$ and using $2^{-\Delta \Delta C T}$ method. The data shown are the mean values $\pm \mathrm{SE}$ of three replicates, and the significance level relative to controls is ${ }^{*} P<0.05,{ }^{* *} P<0.01$.

(Yang et al., 2012; Gao et al., 2014; Li et al., 2015; Yang et al., 2015). The AP2/ERF gene family has not been characterized in $P$. patens and previous data demonstrated that the AP2/ERF genes were the most abundant transcriptional factors in S. caninervis (Gao et al., 2014). To our knowledge the transcriptome-based identification and classification of AP2/ERF family presented here is the report on AP2/ERF family analysis in any moss species. As such it will lay the foundation for further functional analysis of these AP2/ERF genes in S. caninervis and provide the reference for classification of AP2/ERF gene family in other moss species.

\section{ScAP2/ERFs Showed Commonalities Characteristics}

Phylogenetic tree analysis showed the suitability of using the classic AtAP2/ERF-based classification method with moss derived sequence data and provides evidence that the phylogenetic topology of the AP2/ERF family had already been established before the divergence of vascular plants (Mizoi et al., 2012).

The AP2 domain of AP2/ERF genes was reported to be highly conserved among different plant species, although the sequence similarity outside the AP2 domain was very low (Kizis et al., 2001). Similar to other plants, our results showed that the amino acid composition of the AP2 domain was very conservative between Arabidopsis and S. caninervis, and the 4 G, 16 E, 27 W, $28 \mathrm{~L}, 29 \mathrm{G}$ amino acids were completely conserved in all ScERFs and AtERFs. Other amino acid variation can further refine the classification of genes. For example, the motifs "HLG" and "WLG" in the $\beta 3$-sheet can distinguish a Soloist gene from an $E R F$ gene. Interestingly, we found that in $\beta 2$-sheet of AP2 domain of both $S$. caninervis and Arabidopsis, the vast majority of genes shared an "EIR" element, while "EVR" existed only in the A-1 group of DREB genes and "ERK" was specific to the B-6 subfamily genes in this position.

\section{ScAP2/ERFs Showed Unique Characteristics Compared with Arabidopsis}

ScAP2/ERFs showed commonalities as well as unique characteristics as compared with Arabidopsis. Most of ScERF genes can be classified as described above. A small number of ScERF genes form a unique clade (A-5 DREB) but cannot be classified into a subgroup. The AP2/ERF superfamily in moss encompassed all of the Arabidopsis, and the ratio for each subfamily was constant between $S$. caninervis and $P$. patens. However, compared with Arabidopsis, the percentage of ERF subfamily members in the moss species were greatly expanded, and the gene members in the AP2 and RAV subfamilies were reduced. This adjustment of subfamily members may connected with their proposed functions in both development and stress response. The AP2 subfamily is associated with plant development and ERFs play important roles in abiotic stress tolerance regulation. Abundant ERF genes in moss might 
play important roles in abiotic stress tolerance, which is an adaptive evolutionary adaption for plant species growing in adverse environments (Licausi et al., 2013). Genes from different group may have specific motifs. Conserved motifs outside the AP2 domain region may function as a repression or activation domain, such as ERF-associated amphiphilic repression (EAR: DLNxxP) motif (Ohta et al., 2001; Kagale and Rozwadowski, 2011), LWSY motif (Dubouzet et al., 2003; Chen et al., 2008), and DELL motif (Tiwari et al., 2012). In ScERFs, unigene 74743 had the longest ORF and the AP2 domain was located in the end of C-terminus and contained motif 9 (SXSZPISEDSY) which was repeated five times before the AP2 domain (Figure 4). Bioinformatic analysis has failed to identify 'motif 9' in any additional plant protein. Motif 9 is a novel sequence and the function of this motif is unclear. A priority of our future work is the functional analysis of this novel sequence.

\section{Studies on A-5 Type of DREB Proteins Are Rare Which Play Important Roles in Stress Response}

Dehydration-responsive element-binding proteins genes can be divided into the A1-A6 subgroups according to the sequence similarity of AP2 domain. In Arabidopsis, A-1 (DREB1) and A-2 (DREB2) type DREB proteins have been intensively studied and were reported to play important roles in plant response to cold and osmotic stresses (Sakuma et al., 2002). DREB A-1 and DREB A-2 homologous have been identified and functional analyzed in a variety of plants (Sakuma et al., 2006; Qin et al., 2007; Cong et al., 2008; Yang et al., 2011; Mizoi et al., 2013). Despite representing a large proportion of the DREB family, A-5 type of DREB proteins are poorly studied, and the function and stress response mechanisms of A-5 subgroup proteins is still unclear. In Arabidopsis, stress-associated DREB A-5 proteins were the largest subgroup (with 16 gene members; Sakuma et al., 2002), only the RAP2.1 gene was studied in detail (Dong and Liu, 2010). Additional A-5 DREBs were reported in cotton (Huang and Liu, 2006), soybean (Chen et al., 2007, 2009), potato (Bouaziz et al., 2012), Malus sieversii (Zhao et al., 2012), and Halimodendron halodendron (Ma et al., 2015). Based upon the literature, A-5 DREB proteins showed a diversity in gene expression (i.e., stress response) and putative function (i.e., stress tolerance). The A-5 type of $D R E B$ s response to at least one kind of abiotic stress, such as soybean GmDREB3 responds to a single abiotic stress (i.e., low temperature), while GmDREB2 and PpDBF1 in P. patens can respond to drought, salt, cold, and ABA treatments (Chen et al., 2007, 2009; Liu et al., 2007). Moreover, manyA-5 type of DREB proteins have been shown to enhance stress tolerance such as GmDREB2, GmDREB3, and MsDREBA5 (Chen et al., 2007, 2009; Zhao et al., 2012). Interestingly, RAP2.1 in Arabidopsis was reported to negatively regulate drought and cold tolerance in transgenic
Arabidopsis (Dong and Liu, 2010). P. patens has a single A-5 type DREB (PpDBF1) isolated using FDD technology (Liu et al., 2007). In this study, the vast majority of $D R E B$ genes found in $S$. caninervis transcriptome were A-5 type DREBs, and the majority respond to dehydration and/or rehydration treatment. Unlike Arabidopsis and other plants, we postulate that A-5 type DREB proteins in moss species are the dominant regulator for stress response rather than A-1 (DREB1) and A-2(DREB2) proteins. Hence, to better understand the functional mechanism of plant response to stresses, more members of A-5 subgroup DREB proteins needed to be identified and studied.

\section{AUTHOR CONTRIBUTIONS}

DZ planned and designed the study. BG performed the bioinformatic analysis, YL, HY, and XL executed the experiments, generated the tables and figures. XL wrote the manuscript, AW and YW revised the manuscript and especially contributed to the discussion part of the manuscript. All of the authors read and approved the final manuscript.

\section{ACKNOWLEDGMENT}

This research was supported by the NSFC-Xinjiang Youth Talent Joint Fund Project (U1403302), National Natural Science Foundation of China (31500225), Xinjiang Youth Science and Technology Innovation Project (qn2015bs015).

\section{SUPPLEMENTARY MATERIAL}

The Supplementary Material for this article can be found online at: http://journal.frontiersin.org/article/10.3389/fpls.2017.00262/ full\#supplementary-material

\section{FIGURE S1 | Flowchart of identification of AP2/ERF family genes in} Syntrichia caninervis.

FIGURE S2 | Phylogenetic analysis of AP2/ERF family genes in S. caninervis. The gene tree was constructed using neighbor-joining method using 71 ScAP2/ERFs, Poisson model with pairwise deletion. Bootstrap values from 1000 replicates were used to assess the robustness of the tree.

FIGURE S3 | Phylogenetic analysis of AtDREBs and ScERFs. The gene tree was constructed using neighbor-joining method using all 63ScERFs and 57 AtDREBs represented all the subgroups, Poisson model with pairwise deletion. Bootstrap values from 1000 replicates were used to assess the robustness of the tree. The A-3 subfamily genes in Arabidopsis and S. caninervis were marked in red.

FIGURE S4 | Multiple sequence alignments of AP2 domains of AtERFs. One hundred thirty-nine AtERF (including three Soloists) genes were aligned. Black and light gray shading indicate identical and conserved amino acid residues. The complete conserved amino acids residues were marked with asterisk. The three $\beta$-sheets regions and one $\alpha$-helix region were labeled. 


\section{REFERENCES}

Bailey, T. L., Boden, M., Buske, F. A., Frith, M., Grant, C. E., Clementi, L., et al. (2009). MEME SUITE: tools for motif discovery and searching. Nucleic Acids Res. 37, W202-W208. doi: 10.1093/nar/gkp335

Bouaziz, D., Pirrello, J., Ben Amor, H., Hammami, A., Charfeddine, M., Dhieb, A., et al. (2012). Ectopic expression of dehydration responsive element binding proteins (StDREB2) confers higher tolerance to salt stress in potato. Plant Physiol. Biochem. 60, 98-108. doi: 10.1016/j.plaphy.2012.07.029

Chen, J. Q., Meng, X. P., Zhang, Y., Xia, M., and Wang, X. P. (2008). Overexpression of OsDREB genes lead to enhanced drought tolerance in rice. Biotechnol. Lett. 30, 2191-2198. doi: 10.1007/s10529-008-9811-5

Chen, M., Wang, Q. Y., Cheng, X. G., Xu, Z. S., Li, L. C., Ye, X. G., et al. (2007). GmDREB2, a soybean DRE-binding transcription factor, conferred drought and high-salt tolerance in transgenic plants. Biochem. Biophys. Res. Commun. 353, 299-305. doi: 10.1016/j.bbrc.2006.12.027

Chen, M., Xu, Z., Xia, L., Li, L., Cheng, X., Dong, J., et al. (2009). Cold-induced modulation and functional analyses of the DRE-binding transcription factor gene, GmDREB3, in soybean (Glycine max L.). J. Exp. Bot. 60, 121-135. doi: $10.1093 /$ jxb/ern269

Cong, L., Chai, T. Y., and Zhang, Y. X. (2008). Characterization of the novel gene BjDREB1B encoding a DRE-binding transcription factor from Brassica juncea L. Biochem. Biophys. Res. Commun. 371, 702-706. doi: 10.1016/j.bbrc.2008. 04.126

Czechowski, T., Stitt, M., Altmann, T., Udvardi, M. K., and Scheible, W. R. (2005). Genome-wide identification and testing of superior reference genes for transcript normalization in Arabidopsis. Plant Physiol. 139, 5-17. doi: 10.1104/ pp.105.063743

Dong, C. J., and Liu, J. Y. (2010). The Arabidopsis EAR-motif-containing protein RAP2.1 functions as an active transcriptional repressor to keep stress responses under tight control. BMC Plant Biol. 10:47. doi: 10.1186/1471-2229-10-47

Duan, C., Argout, X., Gebelin, V., Summo, M., Dufayard, J. F., Leclercq, J., et al. (2013). Identification of the Hevea brasiliensis AP2/ERF superfamily by RNA sequencing. BMC Genomics 14:30. doi: 10.1186/1471-2164-14-30

Dubouzet, J. G., Sakuma, Y., Ito, Y., Kasuga, M., Dubouzet, E. G., Miura, S., et al. (2003). OsDREB genes in rice, Oryza sativa L., encode transcription activators that function in drought-, high-salt- and cold-responsive gene expression. Plant J. 33, 751-763. doi: 10.1046/j.1365-313X.2003.01661.x

Gao, B., Zhang, D., Li, X., Yang, H., and Wood, A. J. (2014). De novo assembly and characterization of the transcriptome in the desiccationtolerant moss Syntrichia caninervis. BMC Res. Notes 7:490. doi: 10.1186/17560500-7-490

Hiss, M., Laule, O., Meskauskiene, R. M., Arif, M. A., Decker, E. L., Erxleben, A., et al. (2014). Large-scale gene expression profiling data for the model moss Physcomitrella patens aid understanding of developmental progression, culture and stress conditions. Plant J. 79, 530-539. doi: 10.1111/tpj.12572

Huang, B., and Liu, J. Y. (2006). A cotton dehydration responsive element binding protein functions as a transcriptional repressor of DRE-mediated gene expression. Biochem. Biophys. Res. Commun. 343, 1023-1031. doi: 10.1016/j. bbrc.2006.03.016

Huang, P. Y., Catinot, J., and Zimmerli, L. (2016). Ethylene response factors in Arabidopsis immunity. J. Exp. Bot. 67, 1231-1241. doi: 10.1093/jxb/ erv518

Jin, J., Zhang, H., Kong, L., Gao, G., and Luo, J. (2014). PlantTFDB 3.0: a portal for the functional and evolutionary study of plant transcription factors. Nucleic Acids Res. 42, D1182-D1187. doi: 10.1093/nar/gkt1016

Kagale, S., and Rozwadowski, K. (2011). EAR motif-mediated transcriptional repression in plants: an underlying mechanism for epigenetic regulation of gene expression. Epigenetics 6, 141-146. doi: 10.4161/epi.6.2.13627

Kizis, D., Lumbreras, V., and Pages, M. (2001). Role of AP2/EREBP transcription factors in gene regulation during abiotic stress. FEBS Lett. 498, 187-189. doi: 10.1016/S0014-5793(01)02460-7

Lakhwani, D., Pandey, A., Dhar, Y. V., Bag, S. K., Trivedi, P. K., and Asif, M. H. (2016). Genome-wide analysis of the AP2/ERF family in Musa species reveals divergence and neofunctionalisation during evolution. Sci. Rep. 6:18878. doi: $10.1038 /$ srep 18878

Lata, C., and Prasad, M. (2011). Role of DREBs in regulation of abiotic stress responses in plants. J. Exp. Bot. 62, 4731-4748. doi: 10.1093/jxb/err210
Lei, Z. P., He, D. H., Xing, H. Y., Tang, B. S., and Lu, B. X. (2016). Genome-wide comparison of AP2/ERF superfamily genes between Gossypium arboreum and G. raimondii. Genet. Mol. Res. 15:gmr.15038211. doi: 10.4238/gmr.15038211

Letunic, I., Doerks, T., and Bork, P. (2015). SMART: recent updates, new developments and status in 2015. Nucleic Acids Res. 43, D257-D260. doi: 10. 1093/nar/gku949

Li, X., Zhang, D., Li, H., Gao, B., Yang, H., Zhang, Y., et al. (2015). Characterization of reference genes for RT-qPCR in the desert moss Syntrichia caninervis in response to abiotic stress and desiccation/rehydration. Front. Plant Sci. 6:38. doi: $10.3389 /$ fpls.2015.00038

Li, Y., Wang, Z., Xu, T., Tu, W., Liu, C., Zhang, Y., et al. (2010). Reorganization of photosystem II is involved in the rapid photosynthetic recovery of desert moss Syntrichia caninervis upon rehydration. J. Plant Physiol. 167, 1390-1397. doi: 10.1016/j.jplph.2010.05.028

Licausi, F., Giorgi, F. M., Zenoni, S., Osti, F., Pezzotti, M., and Perata, P. (2010). Genomic and transcriptomic analysis of the AP2/ERF superfamily in Vitis vinifera. BMC Genomics 11:719. doi: 10.1186/1471-2164-11-719

Licausi, F., Ohme-Takagi, M., and Perata, P. (2013). APETALA2/Ethylene Responsive Factor (AP2/ERF) transcription factors: mediators of stress responses and developmental programs. New Phytol. 199, 639-649. doi: 10. $1111 /$ nph.12291

Liu, N., Zhong, N. Q., Wang, G. L., Li, L. J., Liu, X. L., He, Y. K., et al. (2007), Cloning and functional characterization of PpDBF1 gene encoding a DREbinding transcription factor from Physcomitrella patens. Planta 226, 827-838. doi: 10.1007/s00425-007-0529-8

Ma, J. T., Yin, C. C., Guo, Q. Q., Zhou, M. L., Wang, Z. L., and Wu, Y. M. (2015). A novel DREB transcription factor from Halimodendron halodendron leads to enhance drought and salt tolerance in Arabidopsis. Biol. Plant. 59, 74-82. doi: 10.1007/s10535-014-0467-9

Marchler-Bauer, A., Derbyshire, M. K., Gonzales, N. R., Lu, S., Chitsaz, F., Geer, L. Y., et al. (2015). CDD: NCBI's conserved domain database. Nucleic Acids Res. 43, D222-D226. doi: 10.1093/nar/gku1221

Mizoi, J., Ohori, T., Moriwaki, T., Kidokoro, S., Todaka, D., Maruyama, K., et al. (2013). GmDREB2A;2, a canonical DEHYDRATION-RESPONSIVE ELEMENT-BINDING PROTEIN2-type transcription factor in soybean, is posttranslationally regulated and mediates dehydration-responsive elementdependent gene expression. Plant Physiol. 161, 346-361. doi: 10.1104/pp.112. 204875

Mizoi, J., Shinozaki, K., and Yamaguchi-Shinozaki, K. (2012). AP2/ERF family transcription factors in plant abiotic stress responses. Biochim. Biophys. Acta 1819, 86-96. doi: 10.1016/j.bbagrm.2011.08.004

Nakano, T., Suzuki, K., Fujimura, T., and Shinshi, H. (2006). Genome-wide analysis of the ERF gene family in Arabidopsis and rice. Plant Physiol. 140, 411-432. doi: $10.1104 / p p .105 .073783$

Narsai, R., Ivanova, A., Ng, S., and Whelan, J. (2010). Defining reference genes in Oryza sativa using organ, development, biotic and abiotic transcriptome datasets. BMC Plant Biol. 10:56. doi: 10.1186/1471-2229-10-56

Ohta, M., Matsui, K., Hiratsu, K., Shinshi, H., and Ohme-Takagi, M. (2001). Repression domains of class II ERF transcriptional repressors share an essential motif for active repression. Plant Cell 13, 1959-1968. doi: 10.1105/tpc.13.8.1959

Oliver, M. J., Mishler, B. D., and Quisenberry, J. E. (1993). Comparative measures of desiccation-tolerance in the Tortula ruralis complex.1. Variation in damage control and repair. Am. J. Bot. 80, 127-136. doi: 10.2307/2445030

Pan, Z., Pitt, W. G., Zhang, Y., Wu, N., Tao, Y., and Truscott, T. T. (2016). The upside-down water collection system of Syntrichia caninervis. Nat. Plants 2:16076. doi: 10.1038/nplants.2016.76

Punta, M., Coggill, P. C., Eberhardt, R. Y., Mistry, J., Tate, J., Boursnell, C., et al. (2012). The Pfam protein families database. Nucleic Acids Res. 40, D290-D301. doi: 10.1093/nar/gkr1065

Qin, F., Kakimoto, M., Sakuma, Y., Maruyama, K., Osakabe, Y., Tran, L. S., et al. (2007). Regulation and functional analysis of $\mathrm{ZmDREB} 2 \mathrm{~A}$ in response to drought and heat stresses in Zea mays L. Plant J. 50, 54-69. doi: 10.1111/j.1365313X.2007.03034.X

Sakuma, Y., Liu, Q., Dubouzet, J. G., Abe, H., Shinozaki, K., and YamaguchiShinozaki, K. (2002). DNA-binding specificity of the ERF/AP2 domain of Arabidopsis DREBs, transcription factors involved in dehydration- and coldinducible gene expression. Biochem. Biophys. Res. Commun. 290, 998-1009. doi: 10.1006/bbrc.2001.6299 
Sakuma, Y., Maruyama, K., Osakabe, Y., Qin, F., Seki, M., Shinozaki, K., et al. (2006). Functional analysis of an Arabidopsis transcription factor, DREB2A, involved in drought-responsive gene expression. Plant Cell 18, 1292-1309. doi: 10.1105/tpc.105.035881

Shu, Y., Liu, Y., Zhang, J., Song, L., and Guo, C. (2015). Genome-wide analysis of the AP2/ERF superfamily genes and their responses to abiotic stress in Medicago truncatula. Front. Plant Sci. 6:1247. doi: 10.3389/fpls.2015.01247

Song, X., Li, Y., and Hou, X. (2013). Genome-wide analysis of the AP2/ERF transcription factor superfamily in Chinese cabbage (Brassica rapa ssp. pekinensis). BMC Genomics 14:573. doi: 10.1186/1471-2164-14-573

Stark, L. R., Mcletchie, D. N., and Mishler, B. D. (2005). Sex expression, plant size, and spatial segregation of the sexes across a stress gradient in the desert moss Syntrichia caninervis. Bryologist 108, 183-193. doi: 10.1639/0007-2745(2005) 108[0183:SEPSAS]2.0.CO;2

Stark, L. R., Nichols, L. II, Mcletchie, D. N., Smith, S. D., and Zundel, C. (2004). Age and sex-specific rates of leaf regeneration in the Mojave Desert moss Syntrichia caninervis. Am. J. Bot. 91, 1-9. doi: 10.3732/ajb.91.1.1

Sun, Z. M., Zhou, M. L., Xiao, X. G., Tang, Y. X., and Wu, Y. M. (2014). Genome-wide analysis of AP2/ERF family genes from Lotus corniculatus shows LcERF054 enhances salt tolerance. Funct. Integr. Genomics 14, 453-466. doi: 10.1007/s10142-014-0372-5

Tamura, K., Peterson, D., Peterson, N., Stecher, G., Nei, M., and Kumar, S. (2011). MEGA5: molecular evolutionary genetics analysis using maximum likelihood, evolutionary distance, and maximum parsimony methods. Mol. Biol. Evol. 28, 2731-2739. doi: 10.1093/molbev/msr121

Tao, Y., and Zhang, Y. M. (2012). Effects of leaf hair points of a desert moss on water retention and dew formation: implications for desiccation tolerance. J. Plant Res. 125, 351-360. doi: 10.1007/s10265-011-0449-3

Tiwari, S. B., Belachew, A., Ma, S. F., Young, M., Ade, J., Shen, Y., et al. (2012). The EDLL motif: a potent plant transcriptional activation domain from AP2/ERF transcription factors. Plant J. 70, 855-865. doi: 10.1111/j.1365-313X.2012. 04935.x

Wood, A. J. (2007). Invited essay: new frontiers in bryology and lichenology The nature and distribution of vegetative desiccation-tolerance in hornworts, liverworts and mosses. Bryologist 110, 163-177. doi: 10.1639/0007-2745(2007) 110[163:IENFIB] 2.0.CO;2

Wu, N., Zhang, Y. M., Downing, A., Zhang, J., and Yang, C. H. (2012). Membrane stability of the desert moss Syntrichia caninervis Mitt. during desiccation and rehydration. J. Bryol. 34, 1-8. doi: 10.1179/1743282011Y.0000000043

Wu, Z. J., Li, X. H., Liu, Z. W., Li, H., Wang, Y. X., and Zhuang, J. (2015). Transcriptome-based discovery of AP2/ERF transcription factors related to temperature stress in tea plant (Camellia sinensis). Funct. Integr. Genomics 15, 741-752. doi: 10.1007/s10142-015-0457-9

Xu, S. J., Jiang, P. A., Wang, Z. W., and Wang, Y. (2009a). Crystal structures and chemical composition of leaf surface wax depositions on the desert moss Syntrichia caninervis. Biochem. Syst. Ecol. 37, 723-730. doi: 10.1016/j.bse.2009. 12.012

Xu, S. J., Liu, C. J., Jiang, P. A., Cai, W. M., and Wang, Y. (2009b). The effects of drying following heat shock exposure of the desert moss Syntrichia caninervis. Sci. Total Environ. 407, 2411-2419. doi: 10.1016/j.scitotenv.2008.12.005

$\mathrm{Xu}, \mathrm{W} ., \mathrm{Li}, \mathrm{F}$., Ling, L., and Liu, A. (2013). Genome-wide survey and expression profiles of the AP2/ERF family in castor bean (Ricinus communis L.). BMC Genomics 14:785. doi: 10.1186/1471-2164-14-785

Xu, Z. S., Chen, M., Li, L. C., and Ma, Y. Z. (2011). Functions and application of the AP2/ERF transcription factor family in crop improvement. J. Integr. Plant Biol. 53, 570-585. doi: 10.1111/j.1744-7909.2011.01062.x

Yang, H., Zhang, D., Li, H., Dong, L., and Lan, H. (2015). Ectopic overexpression of the aldehyde dehydrogenase ALDH21 from Syntrichia caninervis in tobacco confers salt and drought stress tolerance. Plant Physiol. Biochem. 95, 83-91. doi: 10.1016/j.plaphy.2015.07.001
Yang, H., Zhang, D., Wang, J., Wood, A. J., and Zhang, Y. (2012). Molecular cloning of a stress-responsive aldehyde dehydrogenase gene ScALDH21 from the desiccation-tolerant moss Syntrichia caninervis and its responses to different stresses. Mol. Biol. Rep. 39, 2645-2652. doi: 10.1007/s11033-011-1017-6

Yang, W., Liu, X. D., Chi, X. J., Wu, C. A., Li, Y. Z., Song, L. L., et al. (2011). Dwarf apple MbDREB1 enhances plant tolerance to low temperature, drought, and salt stress via both ABA-dependent and ABA-independent pathways. Planta 233, 219-229. doi: 10.1007/s00425-010-1279-6

Yin, B. F., and Zhang, Y. M. (2016). Physiological regulation of Syntrichia caninervis Mitt. in different microhabitats during periods of snow in the Gurbantunggut Desert, northwestern China. J. Plant Physiol. 194, 13-22. doi: 10.1016/j.jplph.2016.01.015

Zhang, G., Chen, M., Chen, X., Xu, Z., Guan, S., Li, L. C., et al. (2008). Phylogeny, gene structures, and expression patterns of the ERF gene family in soybean (Glycine max L.). J. Exp. Bot. 59, 4095-4107. doi: 10.1093/jxb/ern248

Zhang, J., Zhang, Y. M., Downing, A., Cheng, J. H., Zhou, X. B., and Zhang, B. C. (2009). The influence of biological soil crusts on dew deposition in Gurbantunggut Desert, Northwestern China. J. Hydrol. 379, 220-228. doi: 10. 1016/j.jhydrol.2009.09.053

Zhang, J., Zhang, Y. M., Downing, A., Wu, N., and Zhang, B. C. (2011). Photosynthetic and cytological recovery on remoistening Syntrichia caninervis Mitt., a desiccation-tolerant moss from Northwestern China. Photosynthetica 49, 13-20. doi: 10.1007/s11099-011-0002-6

Zhang, Y. M. (2005). The microstructure and formation of biological soil crusts in their early developmental stage. Chin. Sci. Bull. 50, 117-121. doi: 10.1007/ bf02897513

Zhao, X. J., Lei, H. J., Zhao, K., Yuan, H. Z., and Li, T. H. (2012). Isolation and characterization of a dehydration responsive element binding factor MsDREBA5 in Malus sieversii Roem. Sci. Hortic. 142, 212-220. doi: 10.1093/ pcp/pct087

Zheng, Y. P., Xu, M., Zhao, J. C., Zhang, B. C., Bei, S. Q., and Hao, L. H. (2011). Morphological adaptations to drought and reproductive strategy of the moss Syntrichia caninervis in the Gurbantunggut Desert, China. Arid Land Res. Manage. 25, 116-127. doi: 10.1080/15324982.2011.554956

Zhuang, J., Cai, B., Peng, R. H., Zhu, B., Jin, X. F., Xue, Y., et al. (2008). Genomewide analysis of the AP2/ERF gene family in Populus trichocarpa. Biochem. Biophys. Res. Commun. 371, 468-474. doi: 10.1016/j.bbrc.2008.04.087

Zhuang, J., Chen, J. M., Yao, Q. H., Xiong, F., Sun, C. C., Zhou, X. R., et al. (2011). Discovery and expression profile analysis of AP2/ERF family genes from Triticum aestivum. Mol. Biol. Rep. 38, 745-753. doi: 10.1007/s11033-0100162-7

Zhuang, J., Xiong, A. S., Peng, R. H., Gao, F., Zhu, B., Zhang, J., et al. (2010). Analysis of Brassica rapa ESTs: gene discovery and expression patterns of AP2/ERF family genes. Mol. Biol. Rep. 37, 2485-2492. doi: 10.1007/s11033-0099763-4

Zhuang, J., and Zhu, B. (2014). Analysis of Brassica napus ESTs: gene discovery and expression patterns of AP2/ERF-family transcription factors. Mol. Biol. Rep. 41, 45-56. doi: 10.1007/s11033-013-2836-4

Conflict of Interest Statement: The authors declare that the research was conducted in the absence of any commercial or financial relationships that could be construed as a potential conflict of interest.

Copyright (c) 2017 Li, Zhang, Gao, Liang, Yang, Wang and Wood. This is an openaccess article distributed under the terms of the Creative Commons Attribution License (CC BY). The use, distribution or reproduction in other forums is permitted, provided the original author(s) or licensor are credited and that the original publication in this journal is cited, in accordance with accepted academic practice. No use, distribution or reproduction is permitted which does not comply with these terms. 